\title{
Can Security Baselines replace Risk Analysis?
}

R. von Solms

Department of Information Technology

Port Elizabeth Technikon

Private Bag X6011

Port Elizabeth 6000

South Africa

Tel: +27415043604

Fax: +27415043313

email: rossouw@ml.petech.ac.za

\begin{abstract}
To protect the information systems of an organization an appropriate set of security controls need to be installed and managed properly. Many organizations that can afford it conduct either a risk analysis exercise themselves or outsource the process to some consultant. Through such an exercise, the most effective set of controls are recommended. Organizations that cannot afford a risk analysis exercise or cannot conduct it themselves, install controls on an ad hoc basis, with the result that many important business areas might be under protected and vice versa.

Security baselines have provided some guidelines to these organizations on which controls are, under general circumstances, the most effective to install to provide an acceptable level of protection. If an organization requires a higher level of protection in certain areas, a risk analysis can be conducted in those particular areas. As security baselines improve, the need for a further risk analysis will obviously decrease. Will a situation arise where security baselines are so extensive that no need exists for any further risk analysis exercise?
\end{abstract}

\section{Keywords}

Security controls, information security policy, risk analysis, security baselines 


\section{INTRODUCTION}

Risk analysis has traditionally been the dominant technique to identify and assess risk levels within the various business areas in an organization. From this assessment, a set of security controls is proposed to provide adequate security within the different business areas. Unfortunately, this technique is quite complicated and resource intensive, with the result that many organizations do either nothing or bypass risk analysis and propose and implement security controls based on ad hoc thoughts. The result is that many high risk areas might be under protected or vice versa.

According to an information security breach survey (Information security breaches survey, 1996), most small to medium sized organizations do not perform a risk analysis. Possible reasons for this are; firstly, usually most small to medium sized organizations do not have the required expertise to conduct a proper risk analysis and cannot afford the services of a consultant and secondly, the awareness of information security is usually very low at these organizations.

A relative new development has been the establishment of information security baselines, an approach that suggests a minimum set of security controls that should be installed under most circumstances. Thus, any organization can obtain an accepted minimum level of protection that should be adequate under general conditions without going through an expensive risk analysis exercise. This is obviously not the ideal solution, but a lot better than ad hoc approach or doing nothing at all in connection with information security.

The question that will be addressed in the rest of this paper is, to what extent can the clever application of various security baselines, replace risk analysis to a large extent or probably completely.

\section{THE RISK ANALYSIS APPROACH}

Traditional risk analysis is based on a well-defined methodology (Guidelines for Information Security Management, 1996). This methodology basically includes the following steps. Firstly, a boundary is defined for delineating the analysis. All assets are then identified and grouped according to their physical location. Next, all possible threats and vulnerabilities are identified. For each of the threats, a possible rate of occupance is estimated and similar the potential impact is estimated for the loss of each asset. Based on these estimations, risk values are calculated and ordered in descending order. Security controls can now be recommended, based on the risk value and the associated cost. The 'best' controls can now be selected to provide the most cost effective solution. Thus, to determine the most effective set of security controls, a complete analysis is performed to identify and prioritize the various risk areas and to suggest controls to minimize these risks to acceptable levels.

Risk analysis is a very complex, resource intensive process. The main objective of any risk analysis exercise is to recommend a 'best' set of security controls to provide the most appropriate level of information security in the organization. If an organization can identify this set of security controls, using another technique, risk analysis can be abandoned. 


\section{THE SECURITY BASELINE APPROACH}

Security baselines are a well-established concept that won a lot of ground lately. Baselines can be seen as a bottom-up approach, where a generic set of controls is defined for most organizations or business areas under normal circumstances. By installing these baseline controls, an organization can be sure that the most common and serious risks have been addressed adequately under normal, generic circumstances. It must be stressed clearly, that the objectives of security baselines are to provide a minimum level of security.

One of the problems associated with security baseline catalogues is the lack of guidance on which of the controls are applicable to the specific organization or business area under consideration. Such a set of baseline controls address the full information systems environment, from physical security through personnel and logical security. Many of these listed controls might not be applicable to a specific organization, because an organization does not operate in certain areas, for example, if an organization does not allow access to networks by third parties, those controls can be ignored. Baseline catalogues do not provide clear and definite guidelines on how to choose the applicable controls from the set of controls that will provide an acceptable level of security. This can be dangerous as an organization might decide to ignore some controls that were actually required. Traditionally, if an organization decided not to perform a risk analysis, no real acceptable alternative existed to suggest an acceptable set of security controls. Security baselines definitely provide this alternative to identify security controls that cover most risks satisfactory.

\section{RISK ANALYSIS vs SECURITY BASELINES}

Risk analysis is based on a very mathematically sound methodology and certainly the output of a detailed risk analysis exercise is the result of a very thorough process. But, notwithstanding the thoroughness of the methodology, many subjective decisions are made during a risk analysis exercise. For example, decisions must be made whether a threat is of small, medium or large intensity, or a scale of one to five is used to decide on the potential impact of a threat on an asset or group of assets. Thus, although the risk analysis methodology is very sound, the accuracy of the results can be queried, because of possible subjective input decisions into the process.

Many authors doubt the trustworthiness of a risk analysis exercise, because of this reason, for example (Jacobson, 1996), risk analysis is:

- tedious - many decisions to make and lots of data to collect;

- $\quad$ suspect - critics claim results are 'subjective';

- $\quad$ inconsistent - results cannot be repeated;

- $\quad$ useless - senior managers ignore the results;

- in short - painful.

On the other hand, most security baselines available today, are based on either some risk 
analysis exercise performed on a generic environment or alternatively on a general consensus reached between a number of organizations. Thus, by introducing any security baseline will provide nothing more than minimum protection under very general circumstances. Security baselines and risk analysis can supplement each other under certain circumstances. For example, if an organization, introducing some baseline set of controls, identifies any specific threat or an abnormal high potential impact if certain risks materialize, it is definitely advised that a risk analysis is performed in those business areas to ensure that these risks are assessed properly and to propose and implement more stringent controls to provide adequate protection in these high risk areas.

As seen from above, security baselines provide a minimum level of protection, and to provide further protection to some high risk areas or to ensure that some unique situations are covered, a risk analysis is recommended to cover the extraordinary situations, not covered by the baseline set of controls. Obviously, if security baselines cover more and more, previously unique, situations and/or certain high risk business areas, the need for a further risk analysis will decrease. The ultimate solution is obviously if all situations and high risk areas area included in some security baseline and an organization must merely choose the appropriate baseline or set of baselines.

\section{VARIOUS LEVELS OF SECURITY BASELINES}

Many different security baselines are already available to industry. Some baselines are devised for general, discipline independent organizations. Other baselines concentrate more on some specific disciplines. Further, some baselines not only suggest controls for minimum protection, but also for medium-level protection requirements.

One of the better known baselines is probably the Code of Practice for Information Security Management (Code of Practise, 1995). The Code of Practice addresses all types of organizations generically and only aims at minimum protection requirements. It is divided into ten categories, each including a number of proposed controls. Amongst these controls are ten key controls. These ten key controls will always, under all circumstances are applicable to all organizations and need to form part of the set of installed security controls in every organization. The rest of the controls also provide minimum level of protection, but might not always be applicable to every situation. An organization must determine themselves which of these non-key controls are applicable to their situation.

The German IT Baseline Protection Manual [IT Protection Manual, 1996) is a much more comprehensive document. The Baseline Protection Manual addresses low-level up to medium-level protection requirements. Unlike the Code of Practice, the Baseline Protection Manual provides guidance on how to determine specific protection requirements for an organization. It also suggests that if high or very high level of protection is required, a detailed risk analysis should be performed.

Some discipline specific security baselines are beginning to evolve, for example, a security baseline for the medical discipline has already been developed. Many of the environment specific risks, experienced in the medical world, are addressed with this set of baseline controls. Any hospital, for example, introducing this set of baseline controls 
will obviously cover much more risks, more adequately, than by introducing a general baseline, like the Code of Practice.

From these different approaches to baseline security, as discussed above, it can be concluded that security baselines do not provide only minimum security protection any more and further, with more environment or discipline specific baselines appearing, the need to perform a risk analysis, following the introduction of some applicable security baseline, will decrease. Only in really abnormal situations this might still be required.

\section{HIERARCHICAL ORGANIZATION OF SECURITY BASELINES}

Controls proposed in different security baselines cover different protection requirements. These requirements vary from the ten key controls in the Code of Practice, which is the absolute minimum that needs to be installed, to more general controls in the rest of the Code of Practice to controls providing medium-level protection. Discipline specific security baselines can also provide a further level of protection.

If all security baselines can be organized in a hierarchical structure, varying from absolute minimum protection, for example the key controls, to more stringent security requirements many new options arise. An organization can then determine which level of protection is required and if any discipline specific security requirements need to be addressed. Once these decisions have been made, the organization can merely work their way down the hierarchy until the required level of protection is obtained.

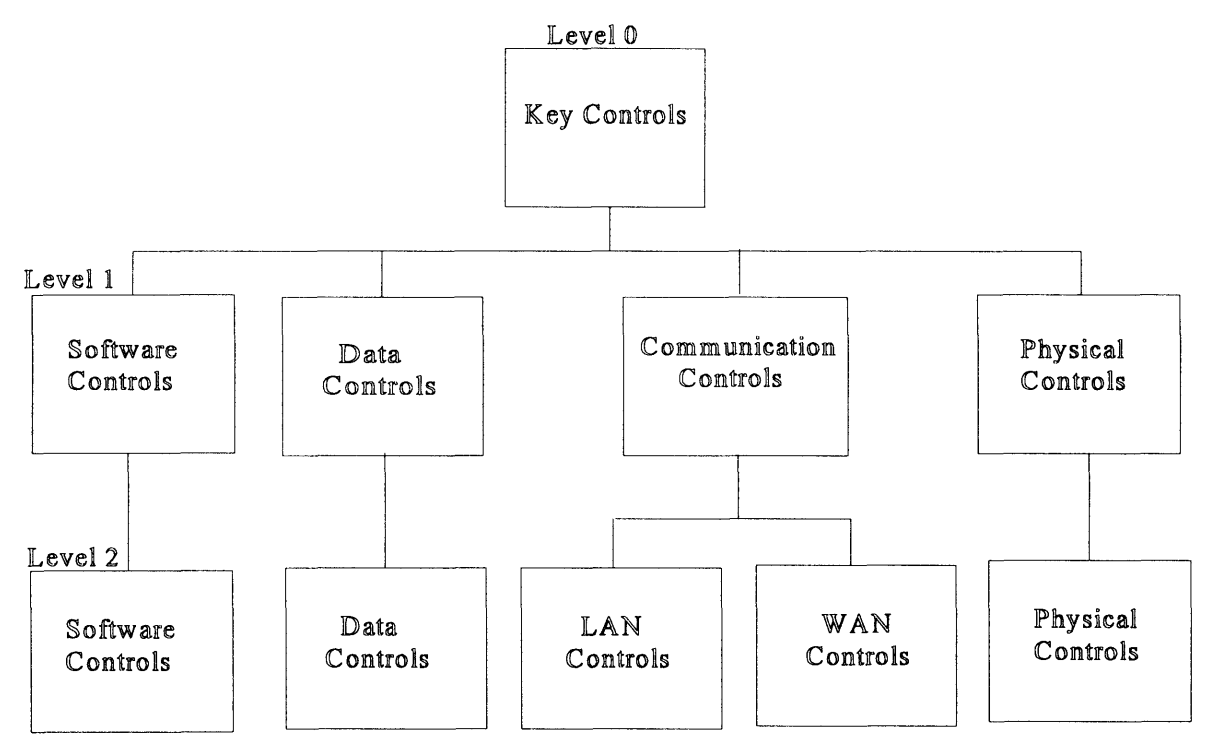

Figure 1 Hierarchical arrangement of security controls 
Figure 1 represents an example of a baseline hierarchy, where the individual controls are grouped under the different categories to be protected.

To include discipline specific controls in the hierarchy, a further dimension can be added to the baseline hierarchy. At a certain level in the hierarchy, a specific area, e.g. physical, may provide various discipline specific alternatives. Figure 2 represents this further dimension graphically.

By integrating various, more stringent security baselines, higher levels of protection can be obtained without having to revert to a risk analysis exercise.

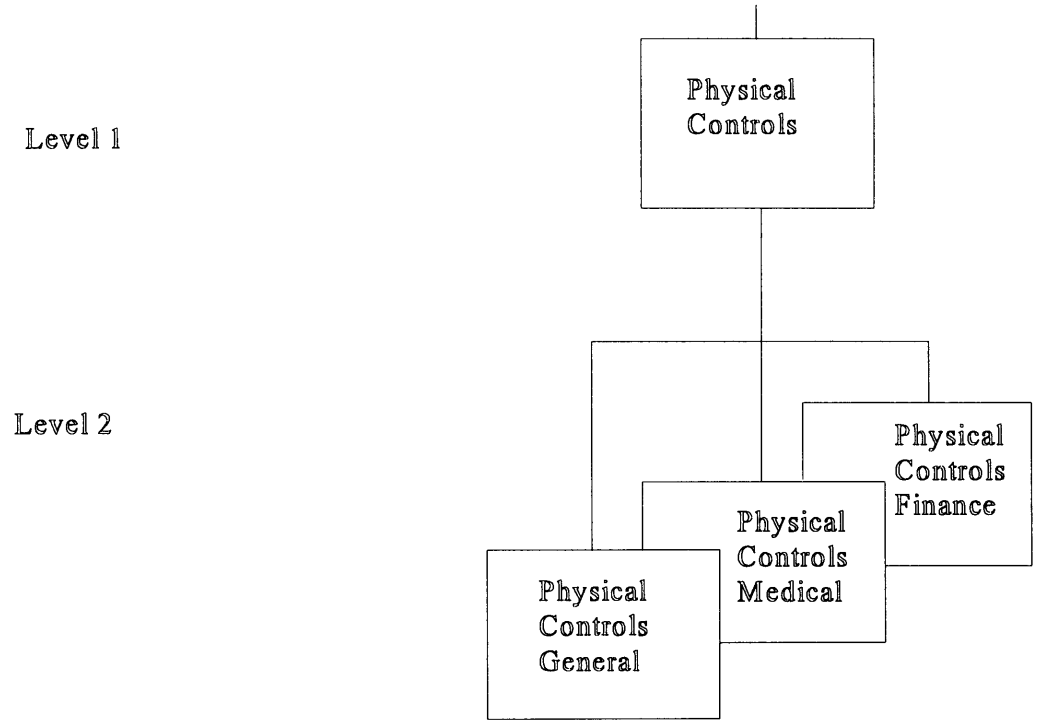

Figure 2 Discipline specific security controls

\section{SEARCH TECHNIQUE THROUGH THE BASELINE HIERARCHY}

As mentioned before, one of the shortcomings in most baseline catalogues is the fact that little guidance is given on which controls need to be selected and will be applicable in a specific situation. If baselines are ordered hierarchically, a definite technique will have to be established to guide the user through the various controls and to determine which controls are applicable and which ones not.

A high level analysis needs to determine which level of the baseline will be appropriate. This high level analysis must consider the business values of all IT systems and the risks from the organization's business point of view.

Once the appropriate level of a security baseline has been determined, a further selection or search technique needs to help the implementor to determine the appropriate controls recommended to obtain the predetermined level of security. Once these controls have been identified, the controls can be installed, managed and monitored. 


\section{CONCLUSION}

All organizations are exposed to many different risks. These risks need to be controlled by the installation of appropriate security controls. To determine the most effective set of controls is not a straightforward exercise. Traditionally, these controls were suggested following a risk analysis exercise. Unfortunately, risk analysis is a very complex, resource intensive exercise and therefore many organizations choose not to perform such an exercise. This is specifically true in the case of small to medium sized organizations. The result is that organizations are either under protected or some ineffective controls are installed.

A security baseline consists of a set of controls that will provide an adequate level of security to most organizations against the general, most common risks. If an organization wishes to install a higher level of security in a specific, very sensitive or important area, a risk analysis can be preformed in that area to address the specific risk situations. As these baselines start to address higher levels of security as well and/or specific discipline environments, the needs for a further risk analysis will decreases. Ultimately, security baselines will be so effective that the need for any further risk analysis will disappear. One prerequisite to the effective implementation of such a technique, is the establishment of an effective search or selection mechanism that can help the user to select an appropriate level of security and the associate controls.

Certainly, such a bottom-up approach will not replace the traditional approach easily. The birth of security baselines has already motivated many more companies to implement security controls, because it is easy to determine which controls need to be installed to provide well-balanced protection. But the intelligent manipulation of security baselines may eliminate the complex, tedious, inconsistent risk analysis exercises completely.

\section{REFERENCES}

Code of Practice for Information Security Management (1995), BS 7799, BSi, UK.

IT Baseline Protection Manual (1996), BSI, Germany.

Guidelines for Information Security Management, Part 3 (1996); PDTR 13335-3, ISO/IEC JTC 1 SC27.

Jacobson R.V. (1996), CORA Cost-of-Risk Analysis, IFIP ‘96 WG 11.2, Samos, Greece.

The Information Security Breaches Survey 1996; NCC, dti, ICL \& UK ITsec 


\section{BIOGRAPHY}

Professor Rossouw von Solms is the head of the department of Information Technology at the Port Elizabeth Technikon since 1989.

Rossouw holds a $\mathrm{PhD}$ from the Rand Afrikaans University in Johannesburg. He is the author of many papers in international journals and have presented a number of papers at international and national conferences. A number of students have completed research qualifications in the area of information security under his leadership.

Rossouw is also the Chairman of Working Group 11.1 of the International Federation for Information Processing (IFIP), that deals with Information Security Management. 